\title{
Vieillissement démographique et participation des personnes âgées au financement des dépenses de santé et des dépenses sociales
}

\section{POPULATION AGING AND THE ELDERLY'S PARTICIPATION IN FUNDING HEALTH AND SOCIAL EXPENDITURES ENVEJECIMIENTO DEMOGRAFICO Y PARTICIPACION DE LAS PERSONAS ANCIANAS AL FINANCIAMIENTO DE LOS GASTOS DE SALUD Y DE LOS GASTOS SOCIALES}

\section{Madeleine Rochon}

Volume 28, numéro 1-2, printemps-automne 1999

L'union libre

URI : https://id.erudit.org/iderudit/010268ar

DOI : https://doi.org/10.7202/010268ar

Aller au sommaire du numéro

Éditeur(s)

Association des démographes du Québec

ISSN

0380-1721 (imprimé)

1705-1495 (numérique)

Découvrir la revue

Citer cet article

Rochon, M. (1999). Vieillissement démographique et participation des personnes âgées au financement des dépenses de santé et des dépenses sociales. Cahiers québécois de démographie, 28(1-2), 299-329.

https://doi.org/10.7202/010268ar
Résumé de l'article

L'apport des personnes âgées au financement des dépenses sociales est loin d'être négligeable dans les pays où ce financement est principalement assuré par les fonds généraux des gouvernements. Au Québec, la contribution moyenne d'une personne âgée atteint ainsi la moitié de celle d'une personne d'âge actif en 1993, et cette contribution est à la hausse. Elle pourrait par contre se stabiliser si les salaires reprenaient leur mouvement de croissance. Lorsqu'on considère cet apport, l'accroissement des charges sociales dû aui vieillissement démographique passe de $19 \%$ à $12 \%$ en 2016, et de $67 \%$ à $44 \%$ en 2041. Si on suppose de plus qu'il progresse (passant de 0,5 à 0,65 fois celui d'une personne d'âge actif), l'accroissement atteint $8 \%$ en 2016 et $35 \%$ en 2041. Les dépenses de santé et de soins de longue durée représentent la moitié de cette augmentation. Outre les changements touchant la nature de ces programmes, leurs coûts unitaires et leur mode de financement, les principaux facteurs qui viendront moduler cette évolution sont le mode d'indexation des transferts publics, la croissance économique, les avoirs financiers des personnes âgées et l'âge à la retraite. 
Cahiers québécois de démographie

Vol. 28, no 1-2, printemps-automne 1999, p. 299-329.

\title{
Vieillissement démographique et participation des personnes âgées au financement des dépenses de santé et des dépenses sociales
}

\author{
Madeleine ROCHON *
}

Les indicateurs classiques visant à apprécier les conséquences du vieillissement démographique pour la capacité de financement des dépenses sociales, notamment l'évolution des rapports de dépendance ou de la charge financière assumée par la population d'âge actif, n'admettent aucun apport des personnes âgées. Or, celui-ci est loin d'être négligeable dans les pays où le financement des programmes sociaux est principalement assuré par les fonds généraux des gouvernements comme c'est le cas en Amérique du Nord et dans une minorité de pays de I'Union européenne. La part du financement gouvernemental des dépenses de protection sociale (vieillesse, santé, emploi et famille) varie en effet fortement d'un pays à l'autre (Lévy, 1997, et Saunier, 1997). En 1994, elle est très faible en France $(23,5 \%), 76,5 \%$ des dépenses y étant assurées par les cotisations sociales, soit 49,1\% par les cotisations des employeurs et $27,4 \%$ par celles des salariés. Elle ne dépasse $50 \%$ que dans les pays anglo-saxons et les pays scandinaves $(80,7 \%$ au

\footnotetext{
Ministère de la Santé et des Services sociaux. L'auteur tient à remercier les personnes suivantes: Madame Sylvie Rheault, économiste à la Direction de l'évaluation et de la recherche du Ministère de la Santé et des Services sociaux, qui a soutenu ses premiers travaux sur la contribution des personnes âgées au financement des dépenses publiques de santé (Rochon, 1994), et Messieurs Jacques Henripin, professeur émérite au Département de démographie de I'Université de Montréal, Jacques Gagnon, professeur à l'Institut de recherche et d'enseignement en fiscalité de l'Université de Sherbrooke, et Claude Montmarquette, professeur au Département de science économique de l'Université de Montréal, qui ont à la fois encouragé ces travaux et suscité leur extension et leur validation.
} 
Danemark). Ainsi, si l'apport des personnes âgées peut paraitre négligeable dans un pays comme la France, il peut être sensible dans des pays comme le Danemark et le Canada, où le financement des dépenses sociales est largement êtatique ${ }^{1}$.

Le Conseil consultatif canadien sur le troisième âge (1991) a souligné la contribution des personnes âgées au financement des dépenses publiques et à l'économie en général. Il a signalé en effet que $8 \%$ de l'ensemble des recettes d'impôts sur le revenu personnel perçues par le Gouvernement fédéral en 1988 provenaient des personnes âgées de 65 ans ou plus (5,4 milliards de dollars) et que ce groupe d'âge représentait $11 \%$ des contribuables. Sans être égale à son poids démographique dans la population adulte $(11 \%$ de la population âgée de 20 ans ou plus), la contribution des personnes âgées au financement des dépenses publiques était donc loin d'être nulle. Les travaux canadiens de projection des coûts des programmes sociaux de Wolfson et Murphy (Murphy et Wolfson, 1992, et Wolfson et Murphy, 1997) tiennent également compte de la participation des personnes âgées aux revenus des gouvernements, car leur modèle prévisionnel inclut leurs principales sources de revenus. Ils ont ainsi établi que cette contribution atteignait $8,2 \%$ des impôts sur le revenu des particuliers et $13,6 \%$ des taxes à la consommation dans l'ensemble du Canada en 1994 (donnée communiquée par M. Wolfson). Les études canadiennes rêcentes portant sur l'équité intergénérationnelle des finances publiques impliquent également la détermination de la contribution de chaque groupe d'âge puisque ce sont les impôts nets, soit les impôts moins les transferts et services reçus, sur la vie du contribuable qui doivent être mesurés par la comptabilité générationnelle (Corak, 1998, et Hicks, 1998).

Le présent article porte sur l'importance actuelle et future de la participation des personnes âgées au financement des dépenses publiques dans le contexte québécois. Il tente de répondre à trois questions : 1) quel est l'apport des personnes âgées au financement public des dépenses de santë et des

1 Les personnes âgées ne contribuent cependant pas au financement des programmes de protection du revenu liê à l'emploi tels l'assurancechômage, rebaptisé assurance-emploi, et le Régime de pensions du Canada (RPC) ou le Régime de rentes du Québec (RRQ). Par contre, une part des revenus de ces deux derniers régimes est capitalisée. De plus, le Gouvernement du Québec a instauré, au moment de la création de la Rêgie de l'assurance-maladie du Québec, un Fonds des services de santé alimenté par les employeurs. 
dépenses sociales lorsqu'on tient compte de l'ensemble des revenus des gouvernements? 2) Comment peut évoluer cet apport dans l'avenir ou, du moins, quels sont les facteurs susceptibles de l'influencer ? Et 3) jusqu'à quel point cet apport peut-il atténuer l'augmentation prévisible du fardeau financier des gouvernements résultant du vieillissement démographique ? Une discussion suivra sur les facteurs économiques et politiques qui peuvent moduler les conséquences du vieillissement démographique.

Les dépenses selon l'âge et le sexe qui servent de point de départ à la projection des coûts portent sur l'année 1991. Bien des modifications de programmes ont eu lieu depuis sous l'impulsion des gouvernements, qui ont voulu freiner la croissance de leurs dépenses et réduire leur déficit, mais des calculs récents indiquent, autant pour les dépenses de santé que pour les dépenses sociales en général, que la structure des coûts selon l'âge est relativement stable. Les calculs sur l'apport des personnes âgées ont été faits sur l'année financière 1993-1994, année de l'introduction d'une nouvelle contribution au Fonds des services de santé. Ce Fonds assure le financement du quart environ des dépenses de santé, et les personnes ayant des revenus qui ne proviennent pas d'un emploi, donc les personnes âgées, sont désormais mises à contribution. L'horizon temporel envisagé pour la projection des coûts peut paraitre lointain bien des changements sociaux, sanitaires, politiques et économiques peuvent survenir d'ici vingt à quarante ans -, mais il permet de saisir la pression que le vieillissement démographique fera subir au système de protection sociale et la manière dont ces changements pourront atténuer ou au contraire accentuer cette pression.

\section{SOURCES DES REVENUS DES GOUVERNEMENTS ET INDICES RELATIFS DE LA CONTRIBUTION DES PERSONNES ÂGÉES}

Pour établir la contribution des personnes âgées au financement des dépenses publiques de santê, il a d'abord fallu déterminer leur participation à chaque poste de recettes du Gouvernement du Québec et du Gouvernement du Canada, en raison de l'importance des transferts fédéraux (en espèces) aux provinces (voir les tableaux $\mathrm{A}$ à $\mathrm{C}$, en annexe). L'indice de la participation relative des personnes âgées prend la forme du rapport entre le montant moyen provenant d'une personne âgée 
(montants fournis par des personnes âgées divisés par la population àgée de 65 ans ou plus) et le montant moyen provenant d'une personne âgée de 20 ans ou plus (montants reçus divisēs par la population âgée de 20 ans ou plus). Cet indice est égal à 1 lorsque la contribution des personnes âgées équivaut à leur fraction dans la population adulte âgée de 20 ans ou plus $(15,5 \%$ en 1993 selon Statistique Canada, 1995). Un second indice sera utilisé dans le calcul de l'évolution de la charge financière résultant du vieillissement démographique, soit le rapport entre la contribution moyenne d'une personne âgée et celle d'une personne d'âge actif (20-64 ans).

\section{Impôt sur le revenu des particuliers}

L'impôt sur le revenu des particuliers représente la plus grande source de revenus des deux paliers de gouvernement: $32,6 \%$ des revenus du Gouvernement du Québec et 44,0\% des revenus du Gouvernement fédêral en 1993-1994 (tableaux A et B). Pour ce poste, l'information est disponible, précise et claire (Ministère du Revenu, 1995: tableau 3, et Revenu Canada, 1995 : tableau de base 4) ${ }^{2}$. On établit la contribution moyenne en divisant le montant global des impôts après crédits ${ }^{3}$ provenant de chaque groupe d'âge par la population de ce groupe d'âge, et non par le nombre de contribuables ${ }^{4}$. La population institutionnalisée est donc comprise (au numérateur et au dénominateur) dans les données fiscales présentées ci-

2 Les données provinciales portent sur toutes les déclarations traitées informatiquement, soit sur 99,3\% d'entre elles, alors que les statistiques fédérales sont produites à partir d'un échantillon stratifié de $1,7 \%$, soit 345685 déclarations en 1993.

3 Au palier provincial, l'impôt après crédits comprend l'impôt à payer (élément 79 , ligne 444 de la déclaration), la surtaxe des particuliers (êlément 82 , ligne 447 de la déclaration) et la contribution au Fonds des services de santé (élément 81 , ligne 446 de la déclaration), moins les crédits suivants : le crédit pour l'entreprise de taxi (élément 90 , ligne 457 de la déclaration), le crédit pour 1hébergement des parents (élément 91 , ligne 458 de la déclaration), le remboursement de la TVQ (élément 92 , ligne 459 de la dêclaration), le remboursement d'impôts fonciers (élêment 93, ligne 460 de la déclaration), le crêdit d'impôt remboursable pour la TVQ (élément 94, ligne 461 de la déclaration) et enfin d'autres crédits (élément 95, ligne 462 de la déclaration). Au palier fédéral, l'impôt après crédits nous est fourni par l'impôt fédéral net (poste 51, ligne 420 de la déclaration).

4 Notons que la proportion de contribuables dans la population est la même, que l'on considère les 20 ans ou plus (85\% en 1993), les 2064 ans $(85 \%)$ ou les 65 ans ou plus $(86 \%)$. 
dessous. Le montant moyen d'impôt d'un Québécois âgé de 65 ans ou plus versé au gouvernement provincial en 1993, ainsi établi, atteint $53 \%$ de celui d'un adulte de 20 ans ou plus ${ }^{5}$, et celle d'un Canadien âgé de 65 ans ou plus au gouvernement fédéral en 1993 correspond à $59 \%$ de celle d'un Canadien âgé de 20 ans ou plus. Cette différence tient, en partie, au fait que l'écart de revenu est moins prononcé au Canada qu'au Québec. En effet, le revenu moyen des personnes âgées atteint $76 \%$ de celui des 20 ans ou plus au Québec mais $80 \%$ dans l'ensemble du Canada selon le recensement de 1991 (tableau 1). Pour cette raison, la contribution des Québécois âgés aux revenus du Gouvernement fédéral a été établie à l'aide des indicateurs québécois (tableau A).

\section{Taxes à la consommation}

Les revenus gouvernementaux provenant des taxes à la consommation (par exemple sur les ventes au détail, le tabac, l'alcool) constituent la deuxième source de revenus en importance, soit environ le cinquième des revenus. Pour ces postes, la contribution des personnes âgées peut être estimée à partir de la consommation déclarée dans les enquêtes (Enquête sur les dépenses des familles, Santé Québec) ${ }^{6}$. L'utilisation de ces enquêtes pose toutefois un problème quand la situation des individus ne peut être distinguée de celle des ménages, ceux-ci étant classés selon l'âge de la personne de référence. Ainsi, dans les données publiées de l'Enquête sur les dépenses des familles de 1992, on ne peut retracer l'information que pour $83,0 \%$ des Canadiens âgés vivant en ménage privé (Statistique Canada, 1994). Cette enquête nous apprend que, comparativement aux mêmes types de ménages, les ménages de personnes âgées consacrent une portion légèrement inférieure de leurs revenus à la consommation courante. Si nous excluons le logement et l'alimentation en incluant les repas pris au restaurant, nous approchant ainsi de l'univers des biens de consommation taxables, la portion du revenu des ménages de

5 Cette proportion résulte du fait que $43 \%$ des personnes âgées ont un impôt après crédits à payer, comparativement à $57 \%$ de la population àgée de 20 ans ou plus, et que le montant moyen d'impôts d'une personne âgée imposable atteint $70 \%$ de celui d'une personne imposable âgée de 20 ans ou plus.

6 Ces enquêtes sont limitées aux ménages privés (Santê Quêbec) ou excluent les pensionnaires d'institutions (Enquête sur les dépenses des familles). 
TABLEAU 1 - Revenu moyen et revenu moyen de placements (en dollars courants), population adulte et population ágée de 65 ans ou plus, Québec et Canada *

\begin{tabular}{|c|c|c|c|}
\hline Revenu & $20+$ & $65+$ & $\begin{array}{l}65+1 \\
20+\end{array}$ \\
\hline
\end{tabular}

Selon le recensement de $1991^{\text {a }}$

Revenu moyen, 1990

\begin{tabular}{llll} 
Canada & 23718 & 19081 & 0,80 \\
Québec & 21541 & 16440 & 0,76 \\
\hline
\end{tabular}

Selon l'EFC, Québec

Revenu moyen

\begin{tabular}{lrrr}
$1991 \mathrm{~b}$ & 21329 & 16244 & 0,76 \\
$1988^{\mathrm{c}}$ & 17364 & 12436 & 0,72 \\
\begin{tabular}{l}
$1988 \mathrm{~b}$ \\
\hline
\end{tabular} & 695 & 1741 & 2,51 \\
\hline
\end{tabular}

Selon les statistiques fiscales de 1993 d, Québec

Revenu moyen total (sans SRG)

$20617 \quad 15294 \quad 0,74$

Revenu moyen de placements

$1575 \quad 3293 \quad 2,10$

- Montant imposable de dividendes de corporations canadiennes

- Intérêts

$432 \quad 1,38$

- Gains en capital imposables

- Autres placements

- Dividendes imposables + gains de capital imposables

- Dividendes imposables $/ 1,25+$ gains de capital imposables / 0,75 e

$\begin{array}{rrr}738 & 2276 & 3,09 \\ 489 & 512 & 1,05 \\ 34 & 73 & 2,15 \\ & & \\ 837 & 1018 & 1,18 \\ & & \\ 899 & 1028 & 1,14\end{array}$

Selon les statistiques fiscales de $1995^{\mathrm{f}}$

Revenu moyen total
Canada
24264
21070
0,87
Québec
21889
17728
0,81

Revenu moyen total sans SRG

Québec

$21642 \quad 16288$

0,75

Sources et note : voir page suivante.

personnes âgées dévolue à la consommation est inférieure dans le cas des ménages d'une personne ( $36 \%$ comparativement à $41 \%$ ) et équivalente dans le cas des ménages composés d'un couple marié avec ou sans enfant ( $42 \%$ et $43 \%)$. Faisant 
Suite du tableau 1

Sources :

a. Revenu moyen recalculé en incluant les personnes n'ayant gagné aucun revenu, Statistique Canada, 1993 : tableau 2.

b. Enquête sur les finances des consommateurs (EFC), dans Fugère, 1994 tableaux 1 et 5 . Les données sont fournies pour la population âgée de 18 ans ou plus (et non de 20 ans ou plus).

c. Enquête sur les finances des consommateurs (EFC), dans Gauthier et Duchesne, 1991; calculs effectués à partir des tableaux 7.1, 7.3, 7.8, $7.10,7.13$ et 7.18 . Les données sont fournies pour la population âgée de 15 ans ou plus (et non de 20 ans ou plus).

d. Les revenus déclarés par ceux qui ont produit une déclaration d'impôt sont reportés à l'ensemble de la population; calculs effectués à partir du tableau 3 de Ministère du Revenu, 1995. Le Supplément de revenu garanti et l'allocation au conjoint (SRG) ne sont pas inclus dans le revenu pour cette année d'imposition.

e. Les montants imposables déclarés sont majorẻs de $25 \%$ clans le premier cas et rêduits de $25 \%$ dans le second.

f. Ministère des Finances, 1997: tableau 3, et Revenu Canada, 1997 : tableau de base 4 .

Note :

* Il s'agit du revenu moyen de l'ensemble de la population et non pas seulement du revenu moyen des personnes qui ont un revenu. Les pensionnaires d'institutions sont exclus, sauf dans les statistiques fiscales.

l'hypothèse que les personnes âgées hors famille ou monoparentales consacrent la même proportion de leur revenu à la consommation que les personnes vivant seules, on peut estimer que les personnes âgées consacrent $39 \%$ de leurs revenus à la consommation courante comparativement à $42 \%$ pour l'ensemble des Canadiens, une proportion inférieure donc de $7 \%$. Compte tenu du fait que le revenu moyen d'un Québécois âgé atteint $81 \%$ de celui d'une personne de 20 ans ou plus (statistiques fiscales de 1993, corrigées pour tenir compte du fait que le Supplément de revenu garanti [SRG] est exclu : tableau 1), la consommation taxable des personnes âgées équivaut à $75 \%$ de celle de l'ensemble de la population. L'indice retenu est donc inférieur à l'indice canadien obtenu par Wolfson et Murphy en 1994, soit 83,4\% (proportion des taxes provenant de personnes âgées, $13,6 \%$, divisée par la proportion de personnes âgées dans la population âgée de 20 ans ou plus, $16,3 \%$ ) mais cohérent par rapport aux différences de revenus des Canadiens et des Québécois âgés (tableau 1). 


\section{Impôt sur le revenu des sociétés et autres revenus gouvernementaux}

Pour les revenus provenant non d'individus mais de sociétés, tel l'impôt sur le revenu des sociétés $(5,4 \%$ et $8,5 \%$ des revenus des gouvernements provincial et fédéral en 19931994), il faut retenir un indicateur de la participation des personnes âgées aux activités commerciales et industrielles des sociétés via les actions qu'elles détiennent. Les enquêtes de même que les statistiques fiscales indiquent qu'en ce qui concerne les revenus provenant de placements de toutes sortes, et notamment les dividendes des corporations canadiennes, le groupe des personnes âgées est en meilleure position que la population plus jeune (tableau 1).

Le meilleur indicateur de la participation relative des personnes âgées aux profits des sociétés nous paraît être le fait qu'elles tirent plus de revenus de dividendes de corporations canadiennes ou de gains en capital que l'ensemble de la population âgée de 20 ans ou plus. Compte tenu du fait que les montants imposables déclarés sont majorés de $25 \%$ dans le premier cas et réduits de $25 \%$ dans le second, l'indice de la contribution des personnes âgées atteint 1,14 (tableau 1). Cependant on peut faire l'hypothèse, assez courante, que la moitié de ces coûts des entreprises sont refilés aux consommateurs $(0,75$ est l'indice retenu pour les taxes à la consommation). Par conséquent, l'indice de la contribution relative des personnes âgées aux impôts des sociétés atteint 0,95 (soit la moitié de la somme de 1,14 et 0,75$)$.

Le même indice $(0,95)$ a été retenu pour $1,1 \%$ des revenus du Gouvernement du Quēbec qui proviennent, par l'intermédiaire de sociêtés d'État, d'activités commerciales qui incluent celles d'organismes publics (permis d'immatriculation pour des vêhicules commerciaux ou institutionnels et vente d'électricité au secteur général et institutionnel). En outre, certains postes de revenus totalisant $8,8 \%$ des recettes du Gouvernement fédéral n'ont pas été répartis, ce qui équivaut à leur attribuer le pourcentage obtenu pour l'ensemble des autres postes de dépenses $(1,1 \%$ de recettes provenant de non-résidents et $7,5 \%$ de recettes non fiscales, dont $5,3 \%$ de produits de placement : tableau A).

Les cotisations des employeurs à l'assurance-emploi (Gouvernement fédéral, tableau A) et au Fonds des services de santé (Gouvernement du Québec, tableau B) sont attribuées à 
la population d'âge actif puisqu'elles sont supposées prélevées sur la masse salariale. De même, la participation des personnes âgées est presque nulle ${ }^{7}$ en ce qui concerne les cotisations des employés à l'assurance-emploi. Cependant, dans le calcul de la contribution des personnes âgées aux revenus du Gouvernement du Québec provenant du Gouvernement fédéral, les cotisations et contributions à l'assurance-emploi ne sont pas considérées puisque celles-ci ne peuvent servir aux transferts aux provinces. L'indice de la participation des personnes âgées aux transferts fédéraux passe ainsi de 0,53 à 0,64 (tableau A).

Les statistiques fiscales provinciales de 1993 indiquent que l'indice de la participation des personnes âgées à la nouvelle contribution des particuliers au Fonds des services de santé est très élevé $(2,53)$ puisque cette contribution s'adresse aux personnes qui ont des revenus ne provenant pas d'un emploi. Les sommes impliquées étant minimes, la contribution des personnes âgées au Fonds des services de santé demeure cependant réduite (indice de 0,13: tableau B).

\section{CONTRIBUTION DES PERSONNES ÂGÉES AU FINANCEMENT DES DÉPENSES PUBLIQUES DE SANTÉ}

Combinés avec les différentes sources de revenus, ces indices nous permettent d'établir la contribution du groupe des personnes âgées au financement des dépenses de santé. L'ensemble des dépenses du ministère de la Santé et des Services sociaux du Gouvernement du Québec est considéré ici ${ }^{8}$. Ces dépenses renvoient à une définition élargie de la santé puisqu'elles incluent notamment tous les soins de réadaptation et de longue durée destinés aux personnes atteintes d'incapacités. Les dépenses retenues s'élèvent à 12,7 milliards de dollars en 1993-1994 (tableau 2). Les revenus sont présumés égaux aux dépenses et cette hypothèse se vérifie quand on omet le service de la dette puisque le total des dépenses du Gouvernement du Québec est alors inférieur à ses revenus.

7 Le taux d'emploi des personnes de 65 ans ou plus n'atteint en effet que $4 \%$ depuis le début des années 1990 (données de 1'Enquête sur la population active transmises par la Direction recherche, ètudes et évaluation de la Sociēté québécoise de développement de la main-d'œuvre).

8 Les dépenses directes du Gouvernement fédéral, qui n'atteignent que $3,4 \%$ des dépenses de santê au Canada en 1991, ne sont donc pas intégrées dans les calculs (Santé Canada, 1996 : tableau 2C). 


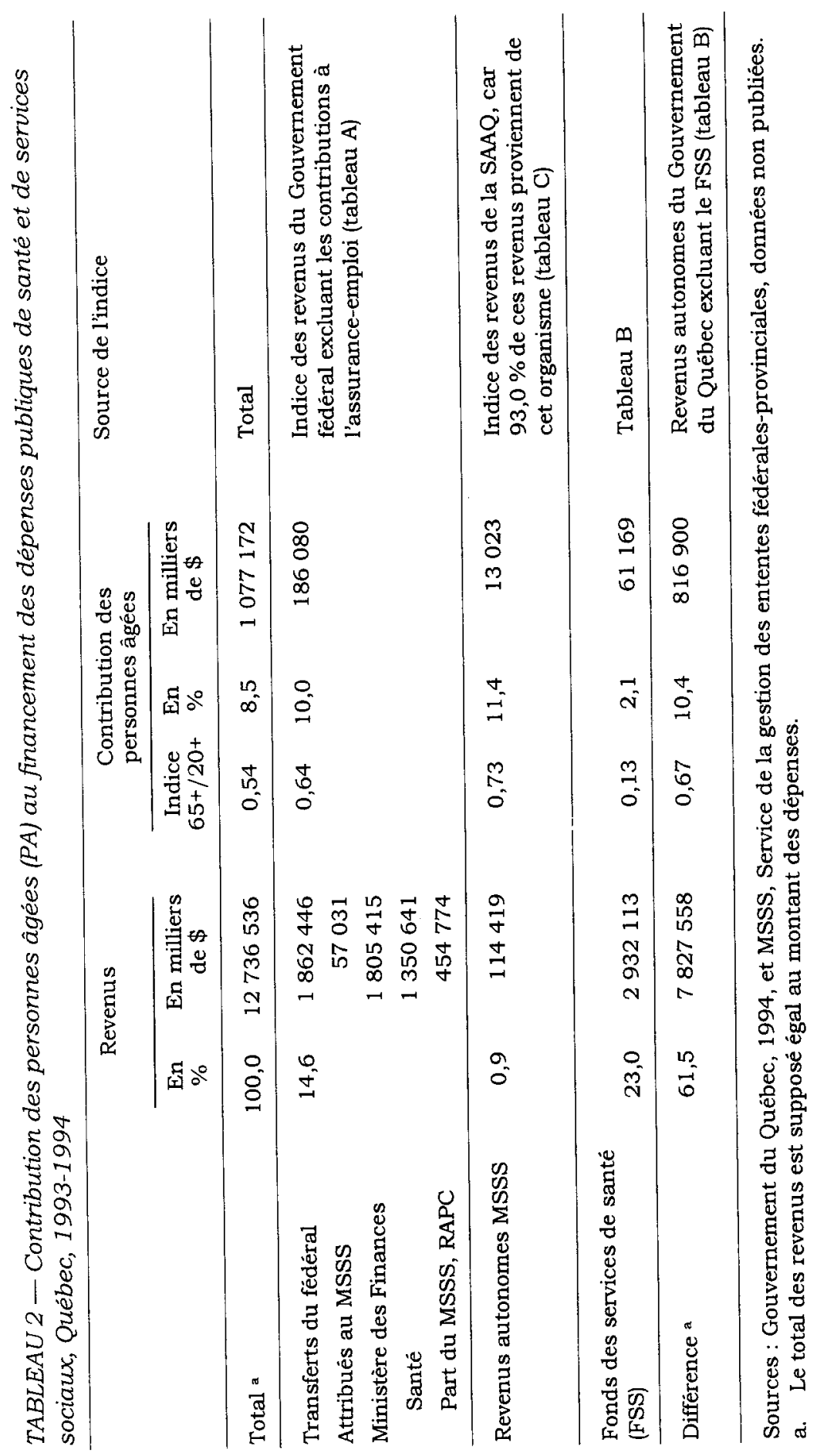


Les cotisations des employeurs et des particuliers au Fonds des services de santé représentent $23 \%$ de ces dépenses, soit 2,9 milliards de dollars (tableau 2). Ce Fonds est destiné au. financement des programmes du régime d'assurance-maladie et des services hospitaliers, et les personnes âgées y participent peu, ne contribuant que pour $2,1 \%$ des cotisations (indice de 0,13 multiplié par la proportion de personnes âgées dans la population âgée de 20 ans ou plus, soit $15,5 \%$ ). Par ailleurs, les transferts fédéraux pour les services de santé et les services sociaux sont estimés à 1,9 milliard de dollars ${ }^{9}$, alors que les revenus autonomes du ministère de la Santé et des Services sociaux n'atteignent que 0,1 milliard de dollars. Les transferts fédéraux assuraient donc près de $15 \%$ des dépenses en 19931994 , les personnes âgées y contribuant pour 10,0 \% (indice de $0,64)$. La différence, soit 7,8 milliards, et donc la plus grande part du financement (61\%), provient des revenus autonomes du Gouvernement du Québec. L'indice de la participation des personnes âgées à ces revenus est le méme que pour les transferts fédéraux $(0,67$ après exclusion du Fonds des services de santé, tableau A).

$\mathrm{Au}$ total, la participation des personnes àgées au financement des dépenses publiques de santé atteint donc 1,1 milliard de dollars en 1993-1994, soit 8,5\% (tableau 2). Ainsi, pour une contribution moyenne égale à 1,00 , celle d'une personne âgée atteint $0,54(8,5 \%$ divisé par $15,5 \%)$ et celle d'une personne âgée de 20 à 64 ans, 1,08 (91,5\% divisé par $84,5 \%$ ). On remarquera que l'indice obtenu $(0,54)$ est pratiquement identique à celui que révèlent les statistiques fiscales provinciales sur l'impôt sur le revenu des particuliers $(0,53$ : tableau B) et que la contribution moyenne d'une personne ägée n'atteint que la moitié de celle d'une personne âgée de 20 à 64 ans.

9 Les informations sur la proportion des dépenses encourues en 1993-1994 par le ministère de la Santé et des Services sociaux dans le cadre des programmes à frais partagés, y compris le Régime d'assistance publique du Canada (RAPC), ont êté obtenues du Service de la gestion des ententes fédérales-provinciales de ce ministère. 


\section{CONTRIBUTION DES PERSONNES ÂGÉES AUX REVENUS GOUVERNEMENTAUX ET AU FINANCEMENT DES DÉPENSES SOCIALES}

Si on s'intéresse non plus seulement au financement des dépenses publiques de santé mais au financement des dépenses publiques en général, alors la participation des personnes âgées semble fort similaire puisque l'indice atteint 0,55 pour l'ensemble des revenus gouvernementaux, estimés à 60 milliards de dollars (tableau 3). Nous avons calculé cet indice en considërant l'ensemble des revenus généraux des gouvernements provincial et fédéral provenant de la population du Québec ${ }^{10}$ ainsi que les cotisations au Régime de rentes du Québec (supposées nulles dans le cas des personnes âgées). On notera que, dans ce calcul également, les dépenses sont supposées égales aux revenus, ce qui se vérifie quand le service de la dette est omis.

Un calcul plus précis quant à certaines dépenses sociales, soit 39,7 milliards de dollars en 1991 selon Gauthier (1995) pour l'éducation, l'aide aux familles, l'aide sociale, l'assurancechoomage, les pensions et rentes aux personnes âgées et la santé et les services sociaux, conduit à un indice similaire mais légèrement inférieur $(0,52)$. La contribution d'une personne âgée atteindrait alors 0,48 fois plutôt que 0,51 fois celle d'une personne d'âge actif.

\section{ÉVOLUTION FUTURE DE LA CONTRIBUTION RELATIVE DES PERSONNES ÂGÉES}

L'indice obtenu étant pratiquement identique à celui que révèlent les statistiques fiscales provinciales sur l'impôt sur le revenu des particuliers, ce dernier semble donner un bon portrait de l'évolution de la contribution relative des personnes âgées aux revenus gouvernementaux. Or la contribution moyenne des personnes âgées aux impôts sur le revenu en proportion de celle de la population d'âge actif s'est élevée de 17 points de pourcentage depuis le début des années 1980 , dont 13 points entre 1985 et 1995 (tableau 4). Le rapport entre

10 Nous avons fixé la part des revenus du Gouvernement fédéral provenant du Québec en utilisant la proportion de la population québécoise dans l'ensemble canadien $(25,0 \%)$. L'indice n'est cependant pas modifié par l'emploi d'une proportion différente, comme la proportion des impôts sur le revenu des particuliers provenant du Québec $(21,6 \%)$. 
TABLEAU 3 - Contribution des personnes âgées $(P A)$ aux revenus gouvernementaux et au financement des programmes sociaux, Québec, 1993-1994

\begin{tabular}{cccc}
$\begin{array}{c}\text { Revenus } \\
\text { en } \\
\text { milliers }\end{array}$ & \multicolumn{2}{c}{ Contribution des pers. âgées } \\
\cline { 2 - 4 } de $\$$ & Indice & En & En milliers \\
& $65+/ 20+$ & $\%$ & de $\$$ \\
\hline
\end{tabular}

Revenus du Gouvernement fédêral 115984000

Revenus du Gouvernement fédéral provenant du Québec *

$\begin{array}{rrrr}28965397 & 0,53 & 8,3 & 2394957 \\ 2776700 & 0,00 & 0,0 & 0\end{array}$

Cotisations au RRQ

700

$\begin{array}{lllll}\text { Revenus autonomes du Gouv. québ. } 28272471 & 0,62 & 9,6 & 2705740 \\ \text { Total : provincial, fédéral et RRQ } & 60014568 & 0,55 & 8,5 & 5100697\end{array}$

Sources : Gouvernement du Canada (1994), Régie des rentes du Québec (1995) et Gouvernement du Québec (1994).

Indice de la contribution par personne âgée comparativement à la contribution par personne âgée de 20 ans ou plus :

- Revenus du Gouvernement fédéral provenant du Québec : tableau A, contributions à l'assurance-emploi incluses.

- Cotisations au Régime de rentes du Québec: contributions minimes des personnes âgées non considérées.

- Revenus autonomes du Gouvernement du Québec : tableau B.

* Proportion des revenus du Gouvernement fédéral provenant du Québec estimée à $25,0 \%$.

TABLEAU 4 - Contribution relative des personnes âgées au financement des dépenses publiques selon les statistiques fiscales du Gouvernement du Québec, Québec

\begin{tabular}{cccccc}
\hline & \multicolumn{2}{c}{$65+/ 20+$ ans } & & $65+/ 20-64$ ans \\
\cline { 5 - 6 } \cline { 5 - 6 } & $\begin{array}{c}\text { Revenu/ } \\
\text { capita * }\end{array}$ & $\begin{array}{c}\text { Impôts après } \\
\text { crédits/ } \\
\text { capita }\end{array}$ & & $\begin{array}{c}\text { Revenu/ } \\
\text { capita * }\end{array}$ & $\begin{array}{c}\text { Impôts après } \\
\text { crédits/ } \\
\text { capita }\end{array}$ \\
\hline 1981 & $57 \%$ & $39 \%$ & & $53 \%$ & $36 \%$ \\
1985 & $67 \%$ & $44 \%$ & & $64 \%$ & $40 \%$ \\
1990 & $71 \%$ & $52 \%$ & & $68 \%$ & $48 \%$ \\
1995 & $81 \%$ & $58 \%$ & $78 \%$ & $53 \%$ \\
\hline
\end{tabular}

Source : Ministère du Revenu et Ministère des Finances, Statistiques fiscales des particuliers.

* Le SRG n'est inclus dans le revenu que depuis 1994. Toutefois aucun impôt n'est payé sur ce revenu. 
la contribution moyenne d'une personne âgée et celle d'une personne d'âge actif est donc sans doute déjà supérieur en $1995(0,53)$ à celui que nous avons établi pour l'année 1993 $(0,51)$.

Cette période un peu particulière n'est cependant pas garante de ce qui se passera dans les prochaines décennies. L'évolution future demeure en effet difficile à prévoir en raison de plusieurs facteurs.

\section{Les facteurs}

Il est vrai que les revenus des Canadiens âgés se sont améliorés dans les dernières années comparativement à ceux des autres groupes d'âge. Selon les données de l'EFC, le revenu moyen - réel, c'est-à-dire tenant compte de l'inflation - des Canadiens âgés a augmenté de $16 \%$ entre 1981 et 1994 alors que celui des Canadiens âgés de 15 à 64 ans est demeuré pratiquement le même (Lindsay, 1997). Au Québec, l'accroissement du revenu moyen durant la même période a été de $21 \%$ dans le cas des personnes âgées, de $5,1 \%$ dans celui des $35-64$ ans et de moins $3,1 \%$ dans celui des 18-34 ans (EFC, compilation RRQ, dans Conseil des Ainés, 1997, tableau 14). Selon cette dernière source, le revenu moyen des Québécois âgés s'établissait à $68 \%$ du revenu moyen de la population âgée de 18 ans ou plus en 1981 mais à $78 \%$ en 1994. L'augmentation des prestations des régimes publics (RPC et RRQ) et privés serait à l'origine de cette hausse des revenus des personnes âgées (Lindsay, 1997). La proportion de personnes âgées qui reçoivent une rente du Régime des rentes du Québec est ainsi passée de $47 \%$ en 1981 à $77 \%$ en 1995 et la proportion de celles qui bénéficient d'un régime privé de $23 \%$ à $33 \%$ (Daigle et Fugère, 1998).

On estime de plus, en France notamment, que le niveau de vie relatif des personnes âgées équivaut désormais à celui des autres groupes d'âge de la population, compte tenu du nombre de personnes par ménage, plus exactement de la composition des ménages (Hourriez et Legris, 1995). Au Québec, cela ne semble cependant pas le cas. Des données similaires indiquent en effet que le niveau de vie des personnes âgées est toujours inférieur à celui de l'ensemble des autres groupes d'âge (Gauthier, Duchesne, Jean, Laroche et Nobert, 1997: tableaux a5.1 et a5.2). En 1995, le revenu total moyen ajusté des unités familiales dont le chef a 65 ans ou plus atteint en 
effet $78 \%$ avant impôt et $87 \%$ après impôt de celui des unités dont le chef a moins de 65 ans et l'écart est encore plus important quand on considère le revenu médian des unités familiales, $71 \%$ avant impôt et $81 \%$ après impôt (données non publiées de l'EFC, compilation du BSQ). Les revenus des personnes âgées sont en effet fortement concentrés au seuil garanti par le SRG, $61 \%$ d'entre elles touchant une prestation de ce programme, les trois quarts de celles qui vivent seules et la moitié des personnes mariées (Daigle et Fugère, 1998). Le Québec est d'ailleurs la province canadienne où la proportion de personnes âgées vivant sous le seuil de faible revenu est la plus élevée (Lindsay, 1997 : tableau 7.12). Même si la proportion de bénéficiaires du RRQ a fortement augmenté, la prestation moyenne demeure en effet trop faible, 4730 dollars en 1995, pour éliminer le recours au SRG; la prestation moyenne de ce programme d'assistance a cependant diminué (Daigle et Fugère, 1998). Rappelons que le régime public de pension (RPC et $R R Q$ ) n'assure que $25 \%$ des gains moyens du cotisant, jusqu'à un montant maximal de 35000 dollars en 1996.

Il est probable que les revenus autonomes moyens des personnes âgées continueront de s'êlever dans l'avenir, et ce au moins jusqu'en 2020. D'une part, les régimes de retraite ne sont pas rendus à maturité (Régie des rentes du Québec, 1996) et, d'autre part, la participation des femmes au marché du travail et le revenu (à âge égal) sont à la hausse dans les générations qui sont les aînés de demain, et ce au moins jusqu'aux premières générations du baby-boom (Gauthier et al., 1997). Cette progression pourrait toutefois être atténuée par la vulné-. rabilité des travailleurs âgés aux récessions économiques, qui semble se traduire par un ralentissement des gains et même par un recul dans les générations les plus récentes d'ainés de 65-69 ans (Firbank, 1997, et Gauthier et al., 1997). Néanmoins, les actifs des régimes de retraite privés, particulièrement sous forme de REER, augmentent fortement. Même s'ils ne profiteront qu'à une minorité de personnes âgées (Wolfson et Murphy, 1997), ces nouveaux revenus seront imposables et. pourront assurer une augmentation de la contribution relative des personnes âgées au financement des programmes publics. ainsi qu'une redistribution intragénérationnelle des revenus plus importante.

Cependant, les transferts publics à l'égard des personnes âgées pourraient diminuer sous l'action de plusieurs facteurs, notamment les modifications aux programmes existants (trans- 
ferts et fiscalité ${ }^{11}$ ), comme il en a été sérieusement question à plusieurs reprises dans le passé récent. D'une part, le programme universel de Pensions de sécurité de la vieillesse (PSV), qui représente le quart des revenus des personnes âgées (le tiers dans le cas des femmes), perdra un peu d'ampleur à cause d'un seuil de récupération partiellement indexé : indice des prix à la consommation (IPC) moins $3 \%$. D'autre part, le programme d'assistance, le SRG, est basé sur le revenu familial. Par conséquent, les revenus accrus des personnes âgées, particulièrement ceux des femmes, viendront se substituer à une partie des transferts publics. De plus, l'évolution récente de la mortalité favorisera un meilleur équilibre des sexes dans la population âgée et entraînera moins de ruptures par veuvage. La proportion des revenus des personnes âgées qui provient des programmes de sécuritê de la vieillesse (PSV et SRG) devrait donc poursuivre sa dêcroissance $(42 \%$ en 1981 comparativement à $35 \%$ en 1995; voir Daigle et Fugère, 1998), mais les nouveaux revenus privés qui se substitueront à ces transferts publics seront imposables.

Un dernier facteur aura toutefois une action capitale sur la contribution relative des personnes âgées en regard de celle des autres groupes d'âge. Il ne suffit pas en effet que les revenus des personnes âgées s'élèvent pour que leur contribution relative augmente. Il faut qu'ils s'élèvent plus rapidement que ceux des autres groupes d'âge. Or, la dernière période a été caractérisée par deux récessions économiques et par la baisse des gains des hommes et des jeunes provenant d'un emploi rémunéré (Picot, 1998). Dans ce contexte, l'indexation des transferts publics était un avantage certain pour les personnes âgées. Toutefois, une reprise de la hausse (réelle) des salaires modifierait cet avantage, toutes les sources de revenus des personnes âgées n'êtant pas pleinement indexées (PSV pour les hauts revenus et plusieurs régimes privés).

La contribution relative des personnes âgées, à taux d'emploi égaux, évoluera donc sous l'action de plusieurs facteurs, les principaux étant l'évolution de la rémunération réelle des

11 L'écart entre le revenu relatif moyen d'une personne âgée $(78 \%$ de celui d'une personne âgée de 20 à 64 ans selon les statistiques fiscales québécoises de 1995) et sa contribution relative aux impôts sur le revenu des particuliers $(53 \%)$ peut paraitre important. Les moyennes cachent cependant des distributions selon le revenu fort différentes selon l'âge. Ainsi, le revenu relatif des personnes âgées paraît moins important quand on considère la médiane plutôt que la moyenne et, de plus, il est fortement concentré dans la classe de 0,50 à 0,75 fois le revenu médian. 
salariés, l'arrivée, pour encore deux décennies, de nouvelles cohortes d'ainés munies de meilleures sources de revenus, du moins pour une partie d'entre elles, et enfin les lois concernant les transferts publics aux personnes âgées et la fiscalité des particuliers. Bien sûr, un renversement de la tendance à l'abaissement de l'âge de la retraite pourrait également avoir un effet important sur la contribution des personnes âgées au financement des dépenses publiques.

\section{Deux hypothèses}

Compte tenu de ces différents éléments, deux hypothèses sur l'évolution de la contribution relative des Québécois âgés au financement public des dépenses de santé et des dépenses sociales sont retenues: une hypothèse de statu quo et une hypothèse d'amélioration. La première ne nie pas l'amélioration. des revenus autonomes de plusieurs groupes de personnes âgées mais suppose que ces gains seront contrebalancés par d'autres facteurs, telle une augmentation des revenus (réels) des plus jeunes. La seconde ne retient comme éventualité que l'amélioration des revenus de source privée des personnes âgées. Dans le premier cas, la contribution moyenne d'une personne âgée atteint la moitié $(0,50)$ de celle d'une personne d'âge actif; dans le second cas, cette contribution est arbitrairement fixée à 0,65 en 2016 . Elle représente donc une hausse de 15 points de pourcentage par rapport à l'hypothèse du statu quo.

\section{Implications pour l'avenir}

La forte augmentation de la charge financière représentée par les dépenses publiques de santé, dans l'hypothèse du maintien des conditions démographiques actuelles ${ }^{12}$ et des dépenses selon l'âge et le sexe ${ }^{13}$, atteint $39 \%$ en 2016 et $123 \%$ en 2041 quand on ne considère que l'apport des 20-64 ans aux

12 La fécondité demeure à 1,6 enfant par femme, la mortalité poursuit sa baisse jusqu'en 2041 et le solde migratoire est modéré, atteignant environ 12000 personnes par an. Les calculs des projections démographiques ont été faits au BSQ à l'aide du modèle de projection provincial. Seul le solde migratoire diffère des hypothèses du scénario de référence A décrit dans Thibault, Gauthier et Létourneau (1996), étant inférieur de moitié.

13 Les dépenses selon l'âge et le sexe établies pour l'année 1991 figurent dans Rochon (1997) pour la santé et dans Gauthier (1995) pour un ensemble de dépenses sociales comprenant ces dépenses de santé. 


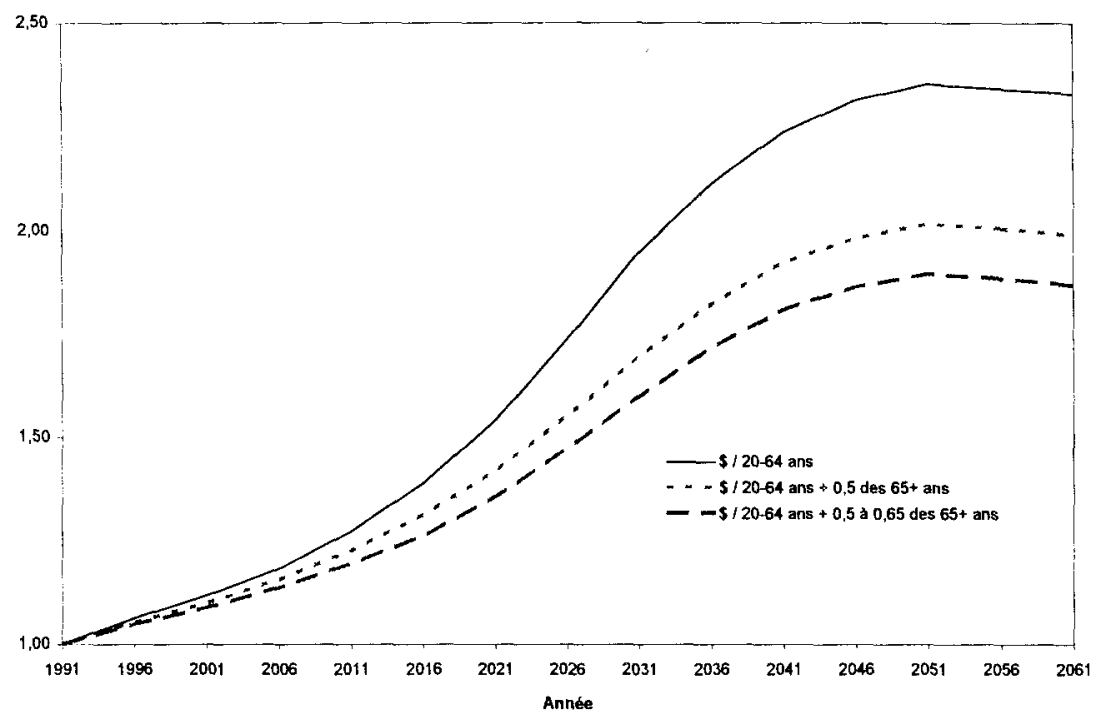

Note : Voir le tableau 5.

FIGURE 1 -Évolution des dépenses de santé en proportion des revenus gouvernementaux, Québec, 1991 à $2061(1991=1,00)$

revenus de l'État (figure 1 et tableau 5). Elle atteint $31 \%$ et $92 \%$ quand on tient compte de la contribution des personnes ãgées et qu'on suppose constante cette dernière. L'accroissement, moins important, de la proportion des revenus gouvernementaux représentée par un ensemble de programmes sociaux (aide aux familles, éducation, aide sociale, assuranceemploi, pensions et rentes, et santë) passe de $19 \%$ à $12 \%$ en 2016 et de $67 \%$ à $44 \%$ en 2041 dans les mêmes conditions (tableau 5). La croissance des dépenses de santé représente la moitié de cette augmentation des charges sociales.

En $1991,11 \%$ de personnes âgées dans la population assumaient $8 \%$ des dépenses publiques de santé et des dépenses sociales en général. En 2041, $28 \%$ de personnes âgées en assumeront $21 \%$. L'augmentation de la part des revenus gouvernementaux provenant des personnes âgées est particulièrement importante entre 2011 et 2031 (figure 1). Elle ne bouge plus après 2036, la croissance du nombre de personnes âgées étant alors pratiquement terminée.

Le poids croissant des personnes âgées n'a donc pas une influence seulement sur le volume des dépenses publiques mais également sur les revenus gouvernementaux. Sans chan- 
TABLEAU 5 -Évolution de la charge financière représentée par les dépenses publiques de santé et un ensemble de dépenses sociales, Québec, 1991, 2016 et $2041(1991=1,00)$

1991- 2016- 1991-

$2016 \quad 20412041$

Dépenses de santé (y compris les soins de longue durée)

Charge par personne d'âge actif : \$/20-64 ans $\quad \begin{array}{llll}1,39 & 1,61 & 2,23\end{array}$

Charge par personne : $\$ / 20-64$ ans $+0,5$ des

$65+$ ans

$1,31 \quad 1,47 \quad 1,92$

Effet de la contribution des personnes âgées

$-6 \%-9 \%-14 \%$

Charge par personne, contribution relative des

PA passe à 0,65 en 2016

$1,26 \quad 1,43 \quad 1,81$

Effet de la contribution des personnes âgées $\quad-9 \%-11 \%-19 \%$

Dépenses sociales (aide aux familles,

éducation, aide sociale, assurance-emploi,

pensions et rentes, santé)

Charge par personne d'âge actif : \$/20-64 ans $\quad 1,19 \quad 1,41 \quad 1,67$

Charge par personne : $\$ / 20-64$ ans $+0,5$ des

$65+$ ans

$1,12 \quad 1,28 \quad 1,44$

Effet de la contribution des personnes âgées $\quad-6 \% \quad-9 \%-14 \%$

Charge par personne, contribution relative des

PA passe à 0,65 en 2016

$1,08 \quad 1,26 \quad 1,35$

Effet de la contribution des personnes âgées

$-9 \%-11 \%-19 \%$

Rapport de dépendance : $65+$ ans/20-64 ans

$1,73 \quad 1,74 \quad 3,00$

Rapport de dépendance : $<20$ et $65+$ ans $/ 20$ -

64 ans

$1,08 \quad 1,36 \quad 1,47$

ger radicalement le portrait de l'évolution de la charge financière, ce facteur, omis dans la plupart des analyses, n'en a pas moins une influence certaine puisque, même constant, il réduit la charge prévue en 2041 de $14 \%$.

Dans l'hypothèse où la contribution relative des personnes âgées continuerait de progresser, de 0,50 en 1991 à 0,65 en 2016 , et demeurerait à cette valeur par la suite, une autre baisse de 5 points de pourcentage serait obtenue. Dans cette perspective, le fardeau prévisible en 2041 serait réduit au total de $19 \%$ et l'apport des personnes âgées représenterait $26 \%$ du 
financement public. La croissance des charges sociales atteindrait alors $8 \%$ en 2016 , au lieu de $19 \%$, et $35 \%$ en 2041 , au lieu de $67 \%$.

L'augmentation prévue par le rapport de dépendance des personnes âgées, celui-ci triple entre 1991 et 2041, est donc loin d'être atteinte puisque les charges sociales s'élèvent de $44 \%$ quand on tient compte de la contribution des personnes âgées, même en la maintenant constante (tableau 5). Le rapport de dépendance général, souvent décrié parce qu'il accorde le même poids aux jeunes et aux personnes âgées ${ }^{14}$, renseigne par ailleurs assez bien sur l'accroissement de ces charges puisqu'il indique une hausse de $47 \%$.

\section{DISCUSSION}

D'autres facteurs démographiques ou sanitaires, tels l'état de santé de la population ou le vieillissement de la population active, ont également une influence sur l'évolution des dépenses ou sur la capacité de la population de les financer. Toutefois, ces transformations ne modifient en rien le fait que les avoirs, la consommation et les revenus des aînés deviendront une source de revenus des gouvernements de plus en plus visible. Cette influence est quasi automatique puisqu'elle ne dépend, essentiellement, que du nombre grandissant de personnes âgées.

À première vue, ces conclusions ne peuvent être étendues à la part que représentent les dépenses publiques dans la production nationale. Ainsi, à productivité égale, la part du PIB québécois représentée par les programmes sociaux, pour moitié transferts en espèces et pour moitié prestations en services (Wolfson et Murphy, 1997, et Gauthier, 1995), pourrait donc augmenter de $67 \%$ au Québec entre 1991 et 2041, soit en moyenne de 1,0\% par année (tableau 5). Le vieillissement est moins prononcé au Canada (22\% de personnes âgées en 2036 comparativement à $28 \%$ au Québec) et l'augmentation de la proportion du revenu national y est moins forte. Elle atteint en effet $31 \%$ entre 1994 et 2036, compte tenu d'une amélioration des taux d'activité féminins et de la maturation du Régime de pension du Canada et du Régime des rentes du Québec (RPC et

14 Selon les données colligées par Gauthier (1995), si les dépenses per capita du groupe d'âge 0-19 ans sont fixées à 1,0, celles du groupe 20-64 ans atteignent 0,4 et celles du groupe 65 ans ou plus 2,1 . 
RRQ), soit $0,6 \%$ par année. L'effet de l'évolution démographique sur la croissance économique demeure cependant matière à débat. Les analyses classiques comme celles de l'OCDE (OCDE, 1996) supposent une désépargne privée, les personnes âgées étant censées consommer une partie de leur patrimoine, alors qu'au contraire les personnes ãgées continuent d'épargner (Du Granrut, 1998, et Denton et Spencer, 1997). De plus, on ne connait pas encore le potentiel de soutien de la croissance de l'économie des avoirs de plus en plus importants des fonds de pension (Du Granrut, 1998). Pour Blanchet (1994), le vieillissement démographique ne pose pas de problème d'accumulation du capital, mais la rêpartition du revenu et du patrimoine entre catégories d'agents économiques pourrait être affectée. Par exemple, la protection accrue des revenus sous forme de régimes privés de retraite ne concerne de façon significative qu'une faible partie de la population (Wolfson et Murphy, 1997), et les transferts privés entre générations (héritage) pourraient être amplifiés, étant répartis entre un plus petit nombre de descendants.

Chose certaine, la croissance économique ou la hausse de la productivité est sans effet sur la moitié des dépenses publiques, soit pour les secteurs de services comme la santé et l'éducation. En effet, la rémunération du personnel y constitue la principale dépense et celle-ci est habituellement majorée en fonction de la hausse générale des salaires. Par conséquent, dans ces secteurs, l'augmentation des coûts unitaires suit la croissance économique. Par contre, la participation à l'enrichissement collectif n'est pas prévue par les programmes canadiens de sécurité du revenu des personnes âgées, qui représentent plus de $20 \%$ des dépenses sociales. La charge financière représentée par ces derniers programmes, les plus touchés par le vieillissement démographique, pourrait donc décliner suite à une croissance économique soutenue. Dans les travaux récents de Wolfson et Murphy (1997) qui portent sur le Canada, une croissance économique de $1 \%$ par année entraîne une baisse, après un peu plus de 40 ans, de $26 \%$ des transferts aux personnes âgées ${ }^{15}$ et de $11 \%$ de l'ensemble des

15 Les droits aux pensions en vertu du principal programme de transfert, le Régime de pensions du Canada (ou le Régime de rentes du Québec), sont indexés sur les salaires mais les pensions, une fois qu'elles commencent à être versées, sont indexées au coût de la vie. Par conséquent, la réduction des charges est moindre pour ce programme. 
charges sociales ${ }^{16}$. Appliquée aux données du Québec, cette baisse de $26 \%$ du coût des pensions entraîne une réduction de $9 \%$ de l'ensemble des charges sociales futures. L'augmentation prévue de leur part du PIB passe alors de $67 \%$ à $52 \%$, la croissance annuelle de $1 \%$ à $0,8 \%$. Une telle progression des salaires rend cependant assez peu probable une augmentation de la contribution relative moyenne des personnes âgées aux revenus gouvernementaux. Dans l'hypothèse d'une contribution relative stable et de la baisse du coût des pensions, la progression des charges sociales québécoises atteindrait $31 \%$ en 2041 (0,5\% par an).

La baisse du poids du vieillissement démographique, qui résulte pour une bonne part de l'allongement de la vie, pourrait certes s'accentuer si les travailleurs âgés étaient maintenus en emploi, comme le recommande fortement l'OCDE (1998). L'élévation de l'âge de la retraite aurait un double effet. Il abaisserait le montant des pensions et augmenterait la production nationale et les entrées fiscales. Ce n'est cependant pas le choix qui a été fait jusqu'ici au Canada, mais l'année 2011, qui correspond à l'année probable du plafonnement de la taille de la population active au Québec, pourrait être favorable au début d'un mouvement dans ce sens.

Jusqu'aux années 1990 , la croissance des dépenses sociales a été importante en raison surtout de leur bonification et de la hausse de la consommation des services publics et de leurs coûts unitaires. Dans la présente décennie, cependant, ce mouvement s'est inversé. Pour éliminer les déficits, non seulement les gouvernements ont réduit leurs dépenses, mais ils ont aussi prévu un financement accru par la hausse des cotisations au RPC et au RRQ de même que l'indexation partielle des tables et crédits d'impôt (IPC-3\%). Wolfson et Murphy (1997) ont déjà montré que la seule indexation partielle des tables et crédits d'impôt est plus que suffisante pour financer le coût du vieillissement démographique au niveau canadien. Oréopoulos et Vaillancourt (1998) ont également montré que le passage d'une tranche d'imposition à une autre qu'entraîne une telle désindexation partielle est suffisante pour égaliser le taux d'imposition net des générations canadiennes présentes et futures, entraînant cependant une hausse de $19,9 \%$ du taux d'imposition net. Ces auteurs arrivent sensi-

16 L'augmentation de la part des dépenses sociales dans la production canadienne passerait alors de $0,6 \%$ à $0,3 \%$ par an. 
blement au même résultat, mais avec une hausse moindre du taux d'imposition net $(15,2 \%)$, lorsqu'ils tiennent compte de l'effet de trois aspects : le mode d'indexation des transferts publics (jointe à une hausse de la productivité de $1 \%$ ), les récentes compressions budgétaires des gouvernements fédéral et provinciaux et enfin les hausses des cotisations au RPC et au RRQ.

Le vieillissement démographique entraîne donc des charges supplémentaires, particulièrement au Québec, où le vieillissement sera fort prononcé, mais cette augmentation pourrait être moins importante que celle qu'on avait initialement prévue. Les décisions politiques concernant la nature des programmes sociaux et leur mode de financement (public ou privé, répartition ou capitalisation, dispositions fiscales...) modulent progressivement le coût de ce vieillissement pour l'ensemble de la société en le répartissant entre les différents groupes sociaux et entre les groupes d'âge. Or, la capacité de payer de chaque groupe évolue, parfois rapidement, sous l'effet de l'interaction de plusieurs facteurs : l'importance et le mode d'indexation des régimes publics et des régimes privés de retraite, les forces du marché du travail, la croissance économique et les transferts privés qui ont lieu au sein des familles. La fiscalité permet de tenir compte de ces évolutions mais certains ajustements seront sans doute nécessaires pour maintenir une certaine équité intra- et intergénérationnelle. L'État doit en effet considérer l'évolution de la capacité financière de chacun dans les efforts d'adaptation de la société aux conséquences du vieillissement démographique.

\section{RÉFÉRENCES BIBLIOGRAPHIQUES}

BLANCHET, Didier. 1994. Les Structures par âge importent-elles? INSEE, Département des études économiques d'ensernble, Division redistribution et politiques sociales, Document de travail G9401, $30 \mathrm{p}$.

CONSEIL CONSULTATIF CANADIEN SUR LE TROISIEME ÂGE. 1991. Les Conditions économiques des aîné-e-s au Canada. Ottawa, Ministère des Approvisionnements et Services Canada, $93 \mathrm{p}$.

CONSEIL DES AÎNÉS. 1997. La Réalité des aînés québécois. Québec, Gouvernement du Québec, 107 p.

CORAK, Miles, éd. 1998. Les Finances publiques et l'équité intergénérationnelle. Ottawa, Statistique Canada, $164 \mathrm{p}$. 
DAIGLE, Élizabeth, et Denis FUGĖRE. 1998. Le Revenu des personnes âgées au Québec en 1995. Régie des rentes du Québec, 29 p.

DENTON, Frank T., et Byron G. SPENCER. 1996. The Changing Economic Circumstances of the Older Population: A Cohort Analysis. Communication présentée au colloque "Le vieillissement des populations revisité ", Montréal, 2-4 octobre.

FUGÈRE, Denis. 1994. L'Évolution du revenu des personnes âgées au Québec entre 1981 et 1991. Québec, Régie des rentes du Québec, $13 \mathrm{p}$.

DU GRANRUT, Charles. 1998. "L'impact du vieillissement démographique. À propos du rapport de l'OCDE ». Futuribles, 228 : 63-68.

FIRBANK, Oscar E.. 1997. "Avance en âge, ressources économiques multiples et situations de vulnérabilité ", Lien social et politiques, 38 : 87-99.

GAUTHIER, Hervé, et Louis DUCHESNE. 1991. Le Vieillissement démographique et les personnes âgées au Québec. Québec, Les Publications du Québec, Bureau de la statistique du Québec, $297 \mathrm{p}$.

GAUTHIER, Hervé. 1995. "Variables dêmographiques et charges sociales: comparaisons annuelles et intergénérationnelles ", Cahiers québécois de démographie, 24, 2 : 285-321.

GAUTHIER, Hervé, Louis DUCHESNE, Sylvie JEAN, Denis LAROCHE et Yves NOBERT. 1997. D'une génération à l'autre: évolution des conditions de vie. Volume 1. Québec, Les Publications du Québec, $257 \mathrm{p}$.

GOUVERNEMENT DU QUÉBEC. 1994. Comptes publics 1993-94. Volume 2. Détail des revenus, crédits et dépenses du Gouvernement du Québec. Québec, Ministère des Finances, 413 p.

GOUVERNEMENT DU CANADA. 1994. Comptes publics 1994. Vol. 1, Revue et états financiers. Ottawa, Approvisionnements et Services Canada.

HICKS, Chantal. 1998. "Le système canadien d'imposition et de transferts. La répartition par groupes d'âge ", dans Miles CORAK, éd. Les Finances publiques et l'équité intergénérationnelle. Ottawa, Statistique Canada : 43-62.

HOURRIEZ, Jean-Michel, et Bernard LEGRIS. 1995. "Le niveau de vie relatif des personnes âgées ", Économie et statistique, 283-284: $137-158$.

LÉVY, Michel Louis. 1997. "Salaires, revenus familiaux, niveaux de vie ", Population et sociétés, 320:1-4.

LINDSAY, Colin. 1997. Un portrait des aînés au Canada. 2e édition. Ottawa, Statistique Canada, no 89-519-XPF au catalogue, 145 p.

MINISTÈRE DES FINANCES. 1997. Statistiques fiscales des particuliers. Année d'imposition 1995. Québec, Les Publications du Québec, $229 \mathrm{p}$. 
MINISTĖRE DU REVENU. 1995. Portrait de la fiscalité des particuliers au Québec. Statistiques 1993. Quêbec, Gouvernement du Quêbec, $237 \mathrm{p}$.

MURPHY, Brian. B., et Michael C. WOLFSON. 1992. "When the babyboom grows old: Impacts on Canada's public sectorn, dans UNITED NATIONS. Changing Population Age Structures. Demographic and Economic Consequences and Implications. Genève, United Nations : 133-147.

OCDE. 1996. Les Enjeux du vieillissement démographique Un défi fondamental pour la politique. Paris, OCDE Poche, no 14, $126 \mathrm{p}$.

OCDE. 1998. Préserver la prospérité dans une sociëté vieillissante. Paris, OCDE, $152 \mathrm{p}$.

OREOPOULOS, Philip, et François VAILLANCOURT. 1998. "L'application au Canada de la méthode de la comptabilité générationnelle: constatations et faussetés", dans Miles CORAK, éd. Les Finances publiques et l'équité intergénérationnelle. Ottawa, Statistique Canada : 9-23.

PICOT, Garnett. 1998. Le Point sur l'inégalité des gains et sur la rémunération des jeunes durant les années 90. Ottawa, Statistique Canada, Direction des études analytiques, Document de recherche no $116,40 \mathrm{p}$.

RÉGIE DES RENTES DU QUÉBEC. 1995. Rapport annuel 1994-1995. Québec, Régie des Rentes du Québec, 72 p.

RÉGIE DES RENTES DU QUÉBEC. 1996. Une réforme du régime des rentes $d u$ Québec pour vous et vos enfants: garantir l'avenir du régime de rentes du Québec. Québec, Régie des Rentes du Québec, $50 \mathrm{p}$.

REVENU CANADA. 1995. Statistiques sur l'impôt des particuliers. Année d'imposition 1993. Ottawa, Ministre du revenu national, $269 \mathrm{p}$.

REVENU CANADA. 1997. Statistiques sur l'impôt des particuliers. Année d'imposition 1993. Ottawa, Ministre du revenu national, $269 \mathrm{p}$.

ROCHON, Madeleine. 1994. Impact des changements démographiques sur l'évolution des dépenses publiques de santé et de services sociaux. Québec, Ministère de la Santé et des Services sociaux, Direction générale de la planification et de l'évaluation, Collection études et analyses, no $21,97 \mathrm{p}$.

ROCHON, Madeleine. 1997. Vieillissement démographique, état de santé et financement des dépenses publiques de santé et de services sociaux. Montréal, Université de Montréal, thèse présentée en vue de l'obtention du grade de Ph.D. en démographie, 212 p. et annexes.

SANTÉ CANADA. 1996. Dépenses nationales de santé au Canada 1975-1994. Rapport sommaire. Ottawa, Santé Canada, Direction générale des politiques et de la consultation. 
SAUNIER, Jean-Marie. 1997. "Les prestations de protection sociale et leur financement 1981-1996 ", Solidarité santé, Études statistiques, no 3 spécial. "Les revenus sociaux": 13-45.

STATISTIQUE CANADA. 1993. Certaines statistiques sur le revenu. Ottawa, Statistique Canada, Recensement du Canada 1991, no 93331 au catalogue, $305 \mathrm{p}$.

STATISTIQUE CANADA. 1994. Dépenses des familles au Canada, 1992. Ottawa, Statistique Canada, no 62-555 au catalogue, 200 p.

STATISTIQUE Canada. Octobre 1995. Estimations annuelles postcensitaires de la population selon l'áge et le sexe pour le Canada, les provinces et les territoires au ler juillet 1993 à 1995. Ottawa, Statistique Canada, Division de la démographie, document polycopié, $4 \mathrm{p}$.

THIBAULT, Normand, Hervé GAUTHIER et Esther LÉTOURNEAU. 1996. Perspectives démographiques : Québec et régions 1991-2041 et MRC 1991-2016. Québec, Les Publications du Québec, Bureau de la statistique du Québec, $439 \mathrm{p}$.

WOLFSON, Michael, et Brian MURPHY. 1997. "Aging and Canada's public sector: Retrospect and prospect", dans K. BANTING et R. BOADWAY, éd. Reform of Retirement Income Policy. Kingston, Queen's University School of Policy Studies : 69-104. 


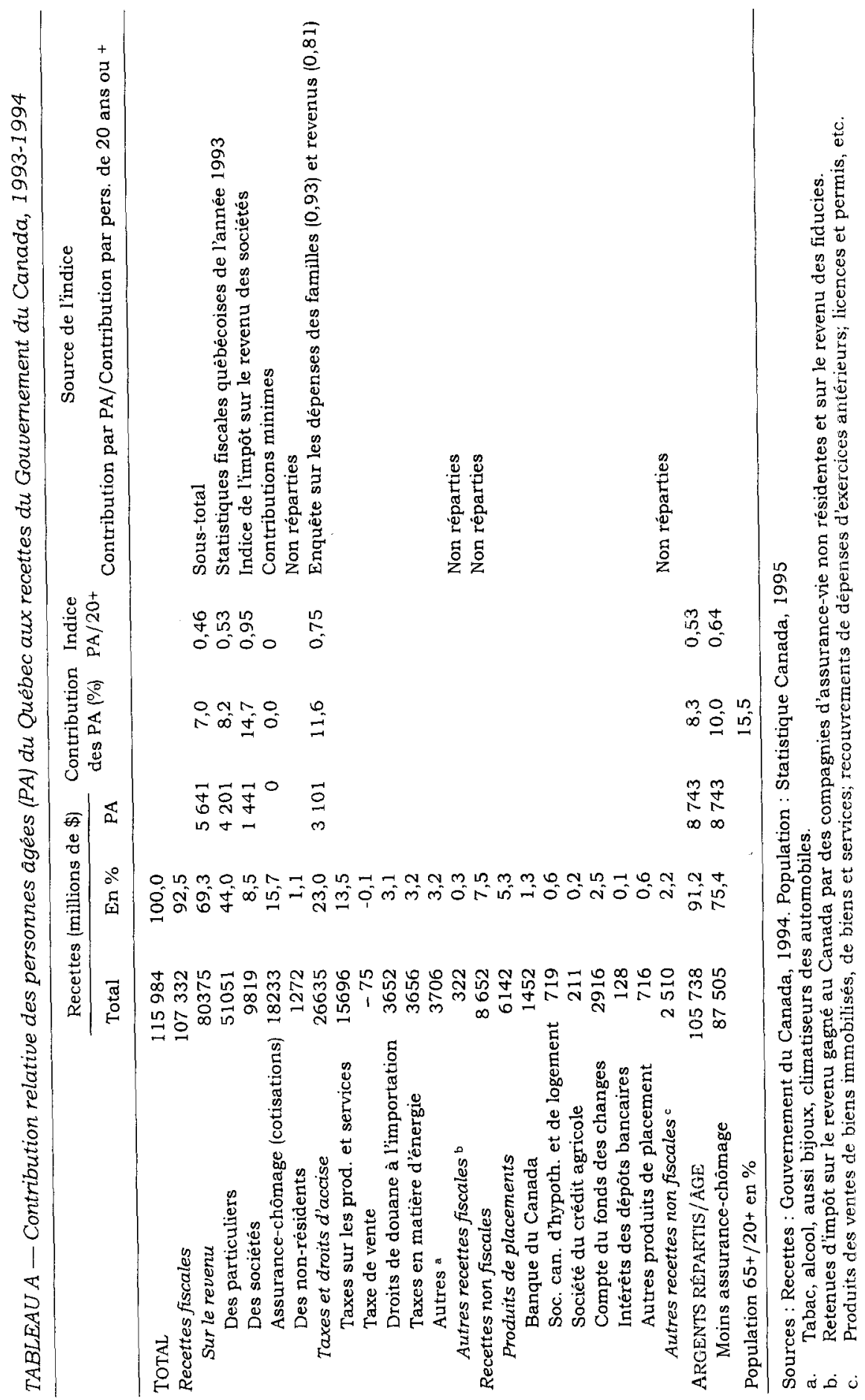




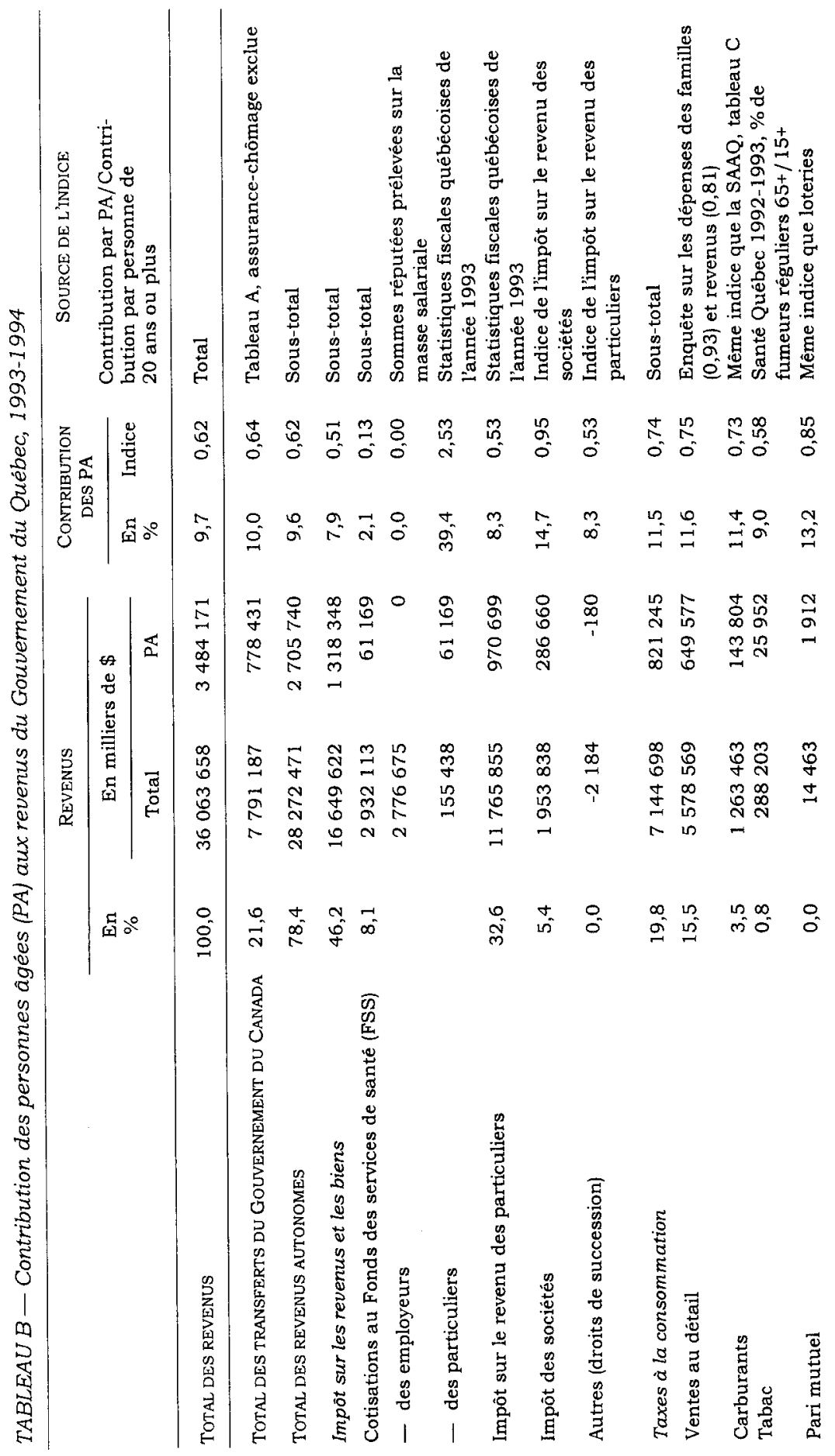




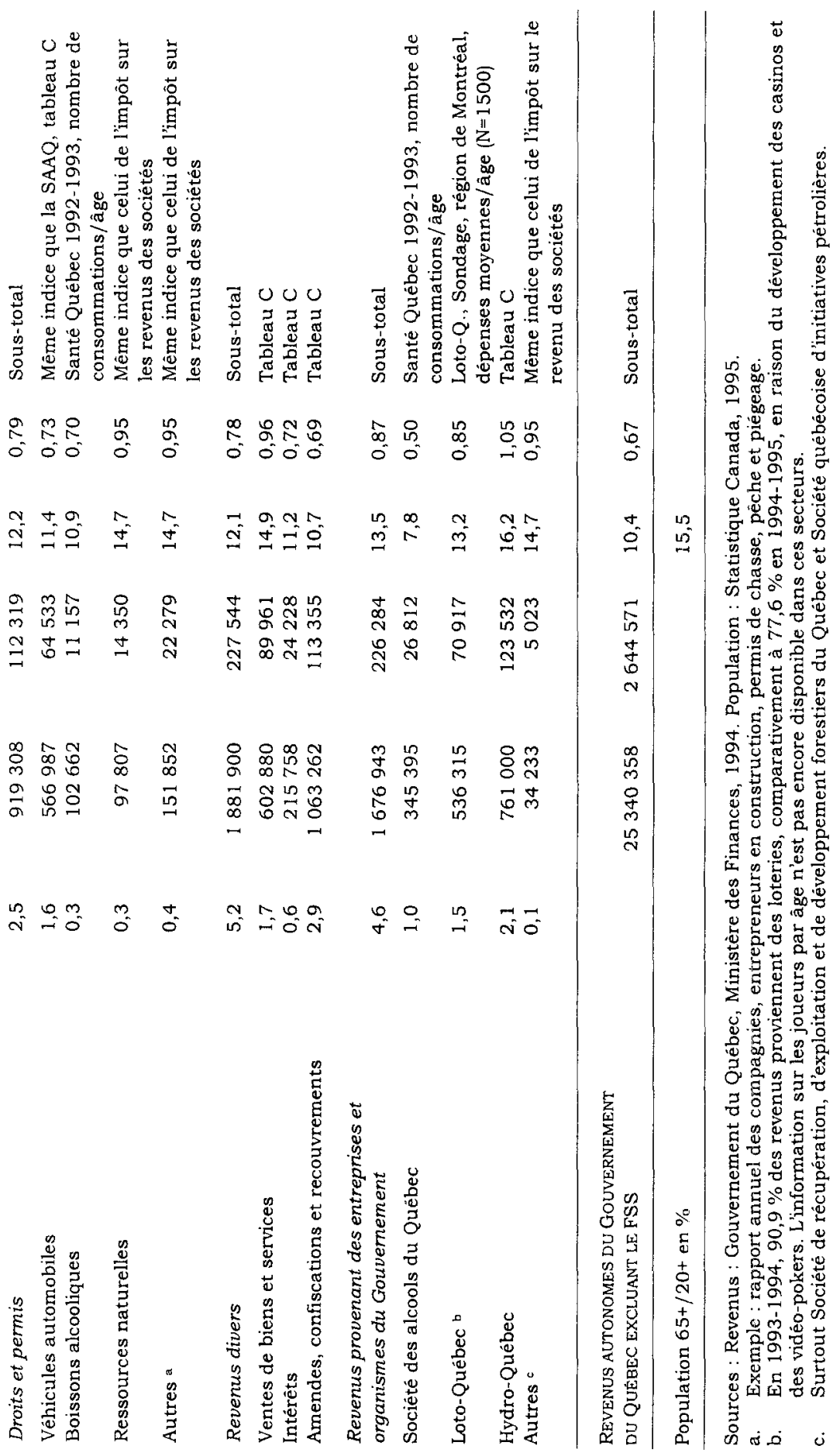




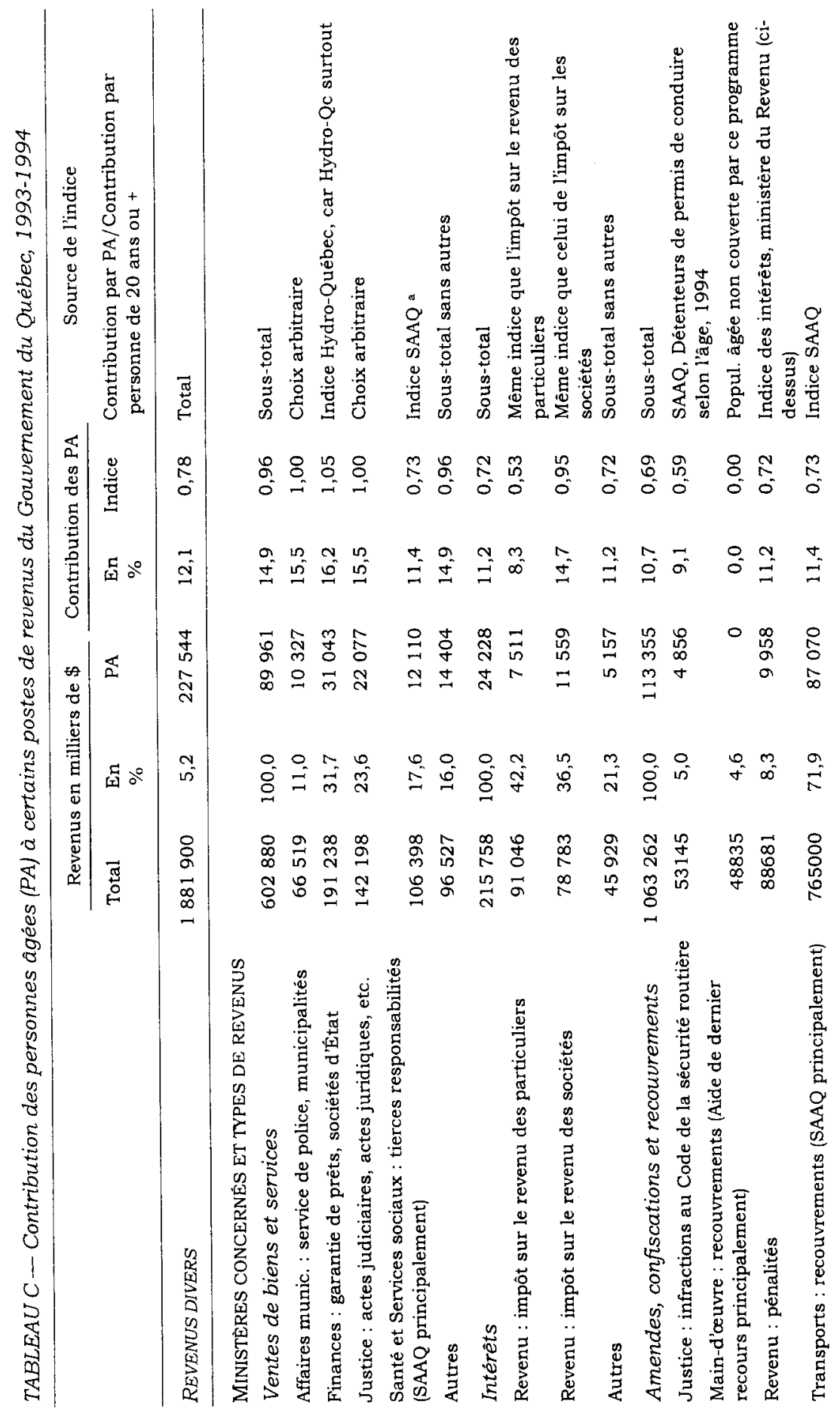




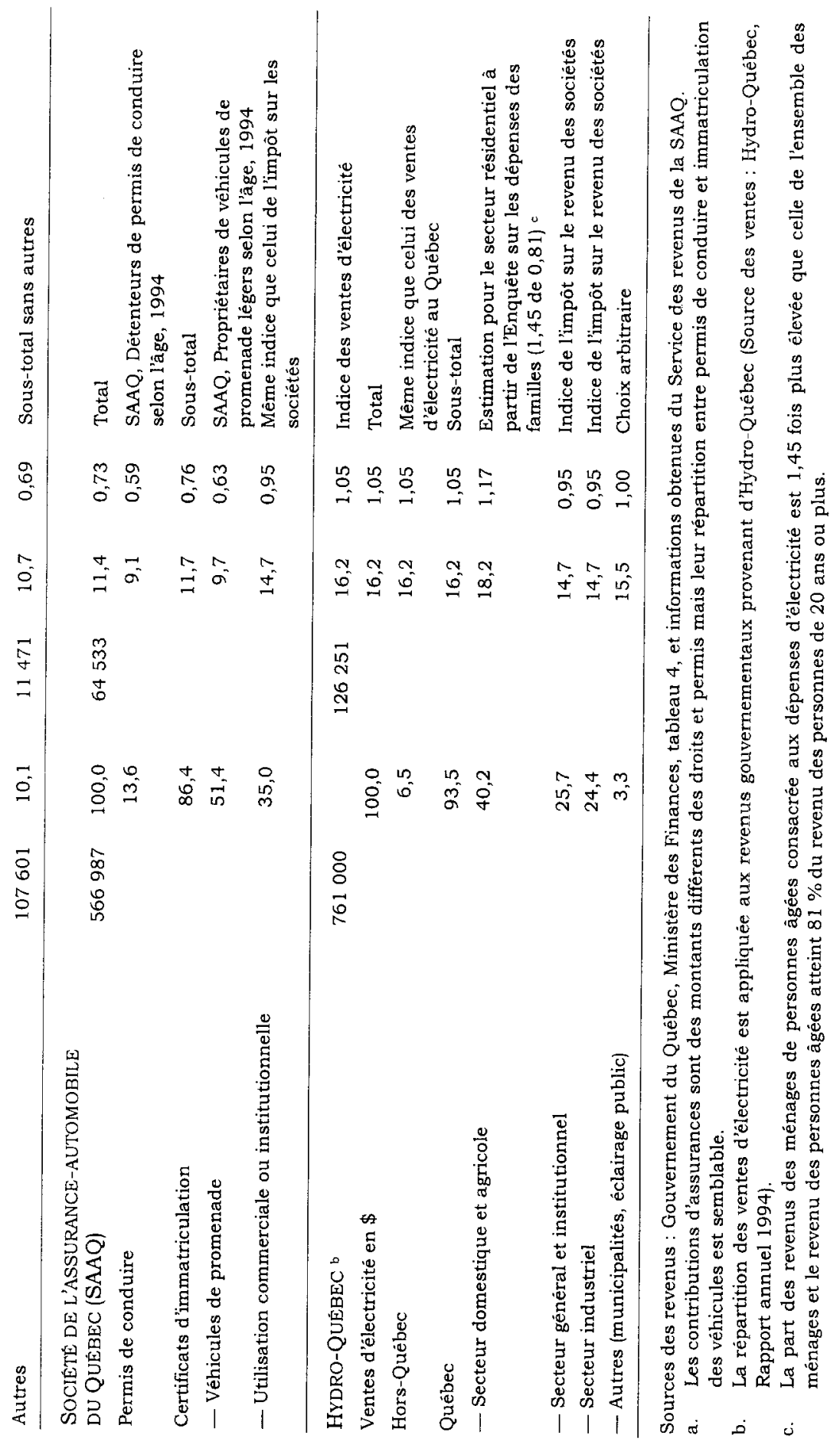

